\title{
Controlled Delivery of Platelet-Derived Proteins Enhances Porcine Wound Healing
}

\author{
Daniel W. Long ${ }^{1,2, \dagger}$, Noah R. Johnson ${ }^{1,2,{ }^{*}, \dagger, \ddagger}$, Eric M. Jeffries ${ }^{1,2}$, \\ Hidetaka Hara $^{3,4, *}$, Yadong Wang ${ }^{1,2,4,5,6,7,{ }^{*}}$ \\ 1. Department of Bioengineering, University of Pittsburgh, Pittsburgh, PA, USA \\ 2. McGowan Institute for Regenerative Medicine, University of Pittsburgh, Pittsburgh, \\ PA, USA \\ 3. Thomas E. Starzl Transplantation Institute, University of Pittsburgh, Pittsburgh, PA, \\ USA \\ 4. Department of Surgery, University of Pittsburgh, Pittsburgh, PA, USA \\ 5. Department of Chemical and Petroleum Engineering, University of Pittsburgh, \\ Pittsburgh, PA, USA \\ 6. Department of Materials Science and Engineering, University of Pittsburgh, \\ Pittsburgh, PA, USA \\ 7. Department of Mechanical Engineering, University of Pittsburgh, Pittsburgh, PA, \\ USA \\ † These authors contributed equally to the current study \\ *Corresponding Author: \\ Yadong Wang, Ph.D. \\ Biomaterials Foundry \\ Department of Bioengineering \\ University of Pittsburgh \\ 3700 O'Hara Street \\ Pittsburgh, PA 15261 \\ Tel: 1-412-624-7196 \\ Email: yaw20@pitt.edu \\ $\ddagger$ Current address: Institute for Neurodegenerative Diseases, University of California, \\ San Francisco, CA, USA \\ ${ }^{¥}$ Current address: Department of Surgery, University of Alabama at Birmingham, \\ Birmingham, AL, USA
}




\section{Abstract}

Platelet-rich plasma (PRP) is widely used for many clinical indications including wound healing due to the high concentrations of growth factors. However, the short half-life of these therapeutic proteins requires multiple large doses, and their efficacy is highly debated among clinicians. Here we report a method of protecting these proteins and releasing them in a controlled manner via a heparin-based coacervate delivery vehicle to improve wound healing in a porcine model. Plateletderived proteins incorporated into the coacervate were protected and slowly released over 3 weeks in vitro. In a porcine model, PRP coacervate significantly accelerated the healing response over 10 days, in part by increasing the rate of wound reepithelialization by $35 \%$ compared to control. Additionally, PRP coacervate doubled the rate of wound contraction compared to all other treatments, including that of free PRP proteins. Wounds treated with PRP coacervate exhibited increased collagen alignment and an advanced state of vascularity compared to control treatments. These results suggest that this preparation of PRP accelerates healing of cutaneous wounds only as a controlled release formulation. The coacervate delivery vehicle is a simple and effective tool to improve the therapeutic efficacy of platelet-derived proteins for wound healing.

Keywords: coacervate; wound healing; coacervate; platelet-rich plasma; polycation; growth factors 


\section{Introduction}

Protein therapies have high potential to improve clinical outcomes in the field of tissue regeneration. These proteins, particularly growth factors and cytokines, relay signals between cells and their extracellular environment, providing cues for cells to proliferate, differentiate, migrate or secrete extracellular matrix (ECM) proteins.

Supplying exogenous growth factors to injured tissues has the potential to accelerate healing and regeneration of tissues; however, they must be applied in a sustained fashion to show efficacy [1-3]. Due to very short half-lives, this is not possible with free growth factors, while large doses are cost-prohibitive and often result in undesirable off-target effects [3]. The ideal regenerative therapy would involve a delivery system to extend the bioactive half-lives of naturally-derived exogenous proteins rather than expensive recombinant proteins. Several approaches have been proposed to meet this clinical need, but few have demonstrated success in a clinical setting [4].

Cutaneous wound healing requires the coordination of many complex processes such as cell proliferation, migration, angiogenesis, and ECM deposition [5]. Each of these processes relies on a specific set of signaling cytokines and growth factors. Wounds are typically able to resolve themselves and close over time, however comorbidities such as diabetes and obesity can impair the healing response and result in a chronic non-healing wound. Chronic wounds are often the result of deficient growth factor signaling or reduced sensitivity to growth factors and require the use of advanced therapies. This contributes to the expanding $\$ 15$ billion market for wound care products in the United States [6]. Even the most advanced wound care options currently available require many treatments and are often unsuccessful, resulting in 185,000 limb amputations performed in the United States each year [6]. 
New approaches addressing the deficient growth factor regulation of chronic wounds are urgently needed.

Growth factors have been assessed for therapeutic benefit in wound applications for more than forty years. Despite thorough evaluation of more than a dozen different growth factors, only human platelet-derived growth factor (PDGF) has received approval for clinical treatment of diabetic ulcers [7]. Under the trade name Regranex, the PDGF gel promotes epithelial cell and fibroblast migration and a meta-analysis of 6 clinical trials reveals that overall the treatment is efficacious [8]. However, this has not necessarily translated to significant clinical usage, in part due to its high cost and poor reputation among clinicians [9]. Other notable candidates that have shown modest results in clinical trials include vascular endothelial growth factor (VEGF) for neuropathic foot ulcers [10], fibroblast growth factor (FGF-1) for deep burns [11], and transforming growth factor-beta 3 (TGF-ß3) for scar prevention [12]. Howeer, none of these have progressed beyond clinical trials, presumably over concerns of a similar fate to Regranex. The multi-faceted etiology of chronic wounds suggests that exogenous application of a single factor may not have a dramatic comprehensive effect on healing outcomes.

One alternative to mono-therapy is to harness the mixture of growth factors produced by the patient themself (autologous) or from a healthy allogenic donor in the form of platelet-rich plasma (PRP). PRP is a fraction of blood plasma containing many therapeutic growth factors released from alpha-granules upon platelet activation [13]. This protein cocktail has high potential to stimulate an accelerated healing response since it contains numerous factors known to play different vital roles in the natural healing progression. Autologous PRP avoids the risk of an immune response during treatment, although allogenic PRP has also been used safely in a 
clinical setting without serious adverse effects as long as the platelets were removed [14]. PRP is currently approved for use in orthopedic applications and is under investigation for several others including wound healing [14-18]. There are many ways to prepare PRP though very few studies have utilized consistent methodology, wound types, or patient demographics, which has led to conflicting data regarding its efficacy. Numerous studies report that PRP improves the wound healing response $[15,18,19]$, while numerous others found it to have no significant effect on healing outcomes [19]. Since each study involves a different formulation, it is difficult to determine the true potential of PRP as a wound healing therapy. As with individual growth factor therapies, the proteins found in PRP also have short half-lives, limiting their efficacy without repeated administrations [3, 18].

Our group has previously described a heparin-based complex coacervate as a protein delivery vehicle [1]. Complex coacervates form when solutions of a cationic polymer and an anionic polymer are mixed, resulting in charge neutralization and phase separation of a polymer-rich phase from the bulk water. Here, we use heparin as the anionic polymer. Many therapeutic growth factors such as VEGF, heparinbinding EGF-like growth factor (HB-EGF), and hepatocyte growth factor (HGF) have a natural affinity for heparan sulfate, a glycosaminoglycan (GAG) found in the ECM [20]. Heparin has similar structure and functionality as heparan sulfate, with the ability to protect growth factors from proteolytic degradation and present them to cell receptors in biomimetic fashion $[20,21]$. A synthetic polycation, poly(ethylene argininylaspartate diglyceride) (PEAD), was developed to interact with the anionic heparin via polyvalent charge attraction. Unlike most other cationic polymers such as polyethylenimine (PEI), PEAD has low cytotoxicity in cell culture and good in vivo biocompatibility [22]. Upon addition to heparin in aqueous solution, PEAD forms a 
complex coacervate containing high concentrations of the polymers while the bulk water phase has little polymer left. Thus this system loads heparin-binding growth factors with high efficiency. The final delivery system consists of coacervate droplets suspended within the aqueous phase. The droplets range from 10 to $500 \mathrm{~nm}$ in diameter and remain stable for at least one month in vivo [1, 23, 24]. Previous studies have shown that this system sustains the release of growth factors for many weeks and significantly extends their half-lives $[1,24-28]$. As demonstrated in several rodent models, coacervate delivery of growth factors can improve cardiac function after myocardial infarction $[23,25,26,29]$ and accelerate wound healing $[24,28,30]$. Other coacervate formulations have shown effective delivery of microRNA inhibitors to fight atherosclerosis [31]. Here we utilize our coacervate system with PRP in a pig model of wound healing. Pig skin possesses very similar architecture and healing mechanisms as human skin and thus provides an appropriate pre-clinical indication for potential success in human patients. Since PRP is widely available and inexpensive, its validation as a growth factor source for therapeutic application in a large animal model brings this technology one step closer to clinical translation.

\section{Materials and Methods}

\section{PRP Preparation and Protein Quantification}

Plasma samples from healthy human patients were obtained from the Central Blood Bank of Pittsburgh. Fresh plasma was obtained within four hours of collection from the patient to maintain high bioactivity of growth factors and cytokines. To isolate PRP, the plasma was then centrifuged at 2,000xg for 15 minutes, and the bottom half of the solution was taken as PRP. Thrombin (Sigma-Aldrich, St. Louis, MO) was added at $1,000 \mathrm{U} / \mathrm{mL}$ and $\mathrm{CaCl}_{2}$ added at $10 \%(\mathrm{w} / \mathrm{v})$ with gentle agitation to activate the platelets for one hour. Platelets released their therapeutic proteins into the 
surrounding plasma upon activation, and the fibrin clot was removed by centrifugation. A 3 kDa MWCO centrifugal filter unit (EMD Millipore, Billerica, MA) was used to further concentrate the proteins in solution. Total protein content was measured by Pierce 660nm protein assay (Thermo Fisher Scientific, Waltham, MA), and individual growth factor concentrations were quantified using sandwich ELISA kits per manufacturer instructions (PeproTech, Rocky Hill, NJ). Concentrated PRP was stored at $-80^{\circ} \mathrm{C}$ until later use.

\section{Platelet-Derived Protein Loading and Release}

The loading and release of platelet-derived proteins from the coacervate delivery system over 4 weeks in vitro was measured. Poly(ethylene argininylaspartate diglyceride) (PEAD) was synthesized as previously described [1]. To form the coacervate, 200uL PRP was combined with 1.6mg heparin (porcine intestine, Scientific Protein Labs, Waunakee, WI), allowing heparin-binding proteins to bind. The coacervate then self-assembled upon the addition of 8mg PEAD in a total volume of $800 \mu \mathrm{L}$.

The coacervate was pelleted by centrifugation at $12,100 \mathrm{xg}$ for 10 minutes and initial growth factor loading was determined by measuring the concentration in the supernatant and comparing to the concentration prior to coacervate formation. Fresh $0.9 \%$ saline was added to resuspend the coacervate and samples were incubated at $37^{\circ} \mathrm{C}$. At predefined time points extending to 21 days, the pelleting procedure was repeated and the supernatant collected for analysis. Fresh saline was then added to resuspend the coacervate.

\section{Animal Care}

All animal procedures were submitted and approved by the University of Pittsburgh's Institutional Animal Care and Use Committee (IACUC) prior to initiation of this study. 
Two 3-month old female Yorkshire pigs were used in this study. The pigs were fed standard lab diet twice per day with unrestricted access to water and their health was monitored at least twice daily for any signs of pain or distress. Following surgeries, pigs were housed individually to avoid perturbation of the wound sites.

\section{Wounding Procedure}

All surgical procedures were conducted under supervision of the Division of Laboratory Animal Resources (DLAR) at the University of Pittsburgh. Sedation was induced using an IM injection of ketamine $(20 \mathrm{mg} / \mathrm{kg})$ and xylazine $(2 \mathrm{mg} / \mathrm{kg})$ and anesthesia was maintained following intubation with 1-3\% isofluorane. Twenty-two full-thickness excisional wounds were created on the back of each pig using $2 \mathrm{~cm}$ diameter biopsy punches to ensure consistent wound size (Shoney Scientific Inc, Waukesha, WI). The punch was driven into the fat layer underlying the dermis, and scissors were used to cut along the underlying fat and remove the skin section. Constant pressure was applied with sterile gauze to stop bleeding, using hemostatic collagen (Davol Inc, Warwick, RI) or epinephrine as needed.

Once the bleeding was stopped, Avitene Ultrafoam (Davol Inc, Warwick, RI) cut to fit the wound was applied, and group-specific treatment solution was added via sterile pipet. Upon addition of these solutions the collagen foam swelled to form a gel and retained the treatments within the wound site. Collagen foams have previously been used in these healing models and are used clinically to facilitate healing [32]. One of four treatments was applied to each wound: (1) saline $(n=9),(2)$ delivery vehicle alone ( $n=9)$, (3) free PRP $(n=8)$, (4) or PRP coacervate $(n=10)$. PRP coacervate was formed by combining $400 \mu \mathrm{l}$ PRP with $3.2 \mathrm{mg}$ filter-sterilized heparin and $16 \mathrm{mg}$ filtersterilized PEAD. Delivery vehicle was unloaded coacervate formed by similar method but with $400 \mu \mathrm{l}$ saline instead of PRP, and free PRP was $400 \mu \mathrm{l}$ PRP in the same total 
volume of saline. Therefore each wound received the same volume of liquid applied to the collagen bandage, and both free PRP and PRP coacervate treated wounds received the same amount of PRP $(400 \mu \mathrm{l}$ or $61.2 \mathrm{mg}$ protein). Wound treatments were assigned by forced randomization to account for any differences in the skin based on location, and each wound was assumed to be independent of other wounds and treatments (Figure 1). All wounds received 5mg ciprofloxacin administered topically to prevent infection. Large Tegaderm bandages (3M, St. Paul, MN) were used to cover wound sites followed by Opsite transparent films (Smith \& Nephew, London, UK) around the perimeter, forming a watertight dressing. A surgical pad sprayed with silicone-based medical adhesive (Hollister Inc, Libertyville, IL) was then applied on top of the entire wound area to protect the wounds and bandages, followed by a custom-fit jacket (Lomir Biomedical Inc, Malone, NY). Baytril (2.5mg/kg) was administered IM once per day for seven days following surgery and amoxicillin $(7 \mathrm{mg} / \mathrm{kg})$ was administered orally twice per day for the remainder of the study to prevent infection. Carprofen $(2 \mathrm{mg} / \mathrm{kg})$ was administered for pain twice daily for five days following surgery. At days 3 and 7, the bandages were changed under brief sedation with ketamine $(20 \mathrm{mg} / \mathrm{kg})$ and xylazine $(2 \mathrm{mg} / \mathrm{kg})$. 
A<smiles>CC(C)(C)CC(O)COCCOCC(O)COC(=O)C(CC(=O)OC(C)(C)C)NC(=O)[C@H](N)CCCNC(=N)N</smiles>

B

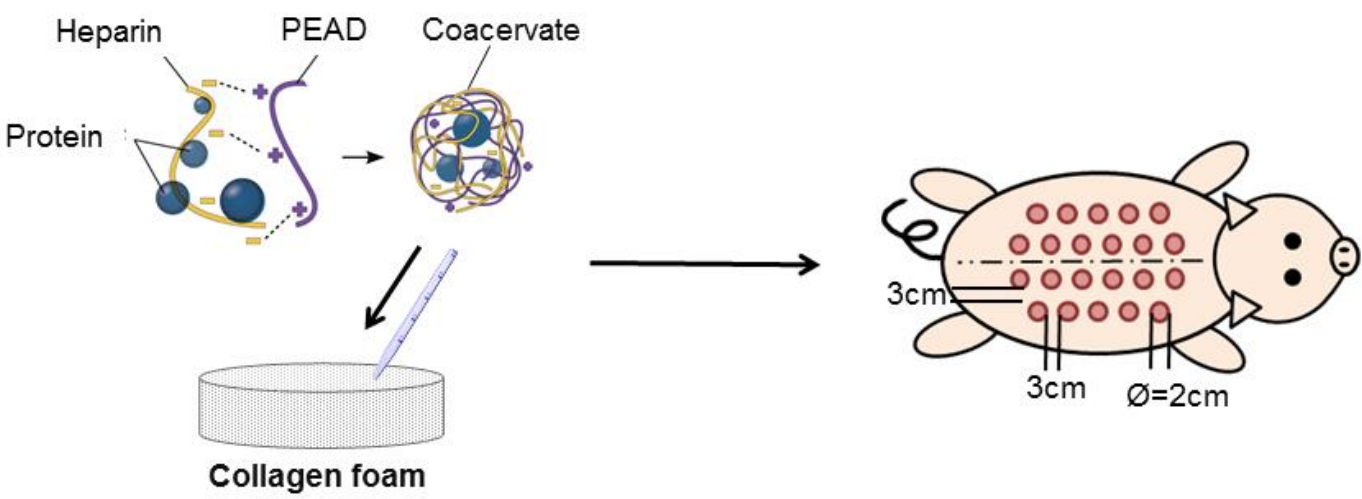

Figure 1. Outline of the wounding procedure

(A) PEAD is synthesized through the conjugation of basic arginine onto a polyester backbone. (B) Combining PEAD with PRP-loaded heparin neutralizes the charged molecules, forming a complex coacervate. Treatments are applied via sterile pipet to a collagen foam before being applied directly to a wound.

Tissue Harvesting and Processing

Ten days after wounding the animals were sacrificed with an overdose of sodium pentobarbital (100mg/kg) administered intravenously. Wounds were photographed for analysis before explant. No signs of infection were present in any wound. The wounds were harvested along with at least $1 \mathrm{~cm}$ of surrounding healthy tissue at the depth of the muscle fascia. Each wound was then cut in half sagittally prior to processing. For histology measurements and immunostaining of cytokeratin, tissues were fixed in $2 \%(\mathrm{w} / \mathrm{v})$ paraformaldehyde for 2 hours and then transferred to a $30 \%$ $(w / v)$ sucrose solution for cryoprotection for 24 hours. Tissues used for 
immunostaining of von Willebrand Factor (vWF) remained unfixed. All tissue samples were then embedded in optimal cutting temperature (OCT) media and frozen in liquid nitrogen-cooled 2-methylbutane. Tissues were then cryosectioned at $6 \mu \mathrm{m}$ thickness for further analysis.

\section{Measuring Wound Contraction}

Images of the wounds were taken during the wounding procedure and after sacrifice. Wound area was measured using an automated filter and measurement macro in Image and compared to the original wound area.

\section{Histology}

Tissue sections were stained with hematoxylin and eosin (H\&E) for gross morphology and qualitative wound healing parameters such as thickness of granulation tissue and the formation of normal dermal substructures. Masson's trichrome stain (MTS) was used to qualitatively assess collagen deposition and alignment within the granulation tissue. Picrosirius red staining was used along with polarized light microscopy to quantify collagen deposition and alignment. Images were taken at the wound edge and center, and the fractional area of collagen was quantified using threshold-based image analysis in ImageJ (NIH). To quantify alignment, the OrientationJ plugin was used to determine the fraction of collagen fibers aligned parallel to the wound surface and the fraction perpendicular to the wound surface. These indicate more and less mature fiber deposition in the scar tissue, respectively, as collagen aligns along the wound surface during scar formation [33]. Collagen alignment is reported as a ratio between parallel and perpendicular-aligned fibers, with higher values indicating an advanced stage of healing. 


\section{Immunostaining Tissue Sections}

Immunofluorescent staining of tissue sections was used to determine the effect of each treatment on angiogenesis and reepithelialization. A rabbit polyclonal von Willebrand Factor (VWF) antibody (1:400 dilution, US Abcam, Cambridge, MA) followed by an Alexa Fluor 594 goat anti-rabbit antibody (Invitrogen, Carlsbad, CA) was used to detect endothelial cells within the tissue. Since healing is delayed in the center of the wound, images were taken at both the wound edge and the center for quantification. The number of vWF+ cells was counted automatically using NIS Elements software (Nikon, Tokyo, Japan) and is reported as blood vessels per $\mathrm{mm}^{2}$ area.

Reepithelialization of a wound reestablishes a functional barrier between the wound and its environment and is essential in preventing infection of the underlying tissue. Reepethelialization was quantified using a rabbit polyclonal cytokeratin antibody (1:100 dilution, US Abcam, Cambridge, MA) followed by an Alexa Fluor 594 goat anti-rabbit antibody. The length of the epidermal tongue was measured and reported as a percentage of the total wound length. Wounds with a damaged epithelial layer were not quantified in this analysis. All images were taken using a Nikon Eclipse Ti inverted microscope.

Statistics and power analysis

All parameters were tested for significant differences between treatment groups using one-way independent analysis of variance (ANOVA) followed by Gabriel's post hoc testing with a significance value $p<0.05$. Analysis was performed using SPSS 22.0 software. Sample size was determined through a power analysis in $\mathrm{G}^{*}$ Power, using wound closure rates reported in our previous wound healing study [24]. This analysis 
showed a sample size of at least $n=4$ wounds per treatment group is needed to gain $80 \%$ power.

\section{Results}

Coacervate system preferentially loads and releases heparin-binding growth factors PEAD carries two positive charges per repeat unit. The polycation forms a complex coacervate when mixed with anionic heparin, visible as a turbid solution. The natural affinity between many therapeutic growth factors and heparin allows these proteins to preferentially load into the coacervate system. Although only $7 \%$ of total PRP proteins were loaded, heparin-binding VEGF and PDGF had loading efficiencies exceeding $60 \%$ (Figure 2a). These proteins exhibited a burst release in the first day, followed by a nearly linear release over the following three weeks (Figure 2b). 
A

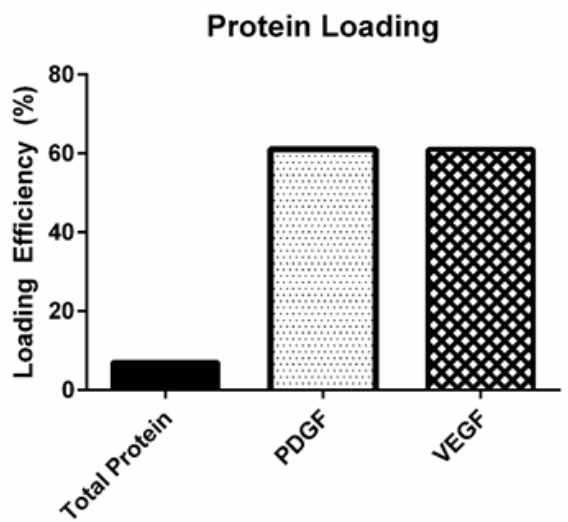

B

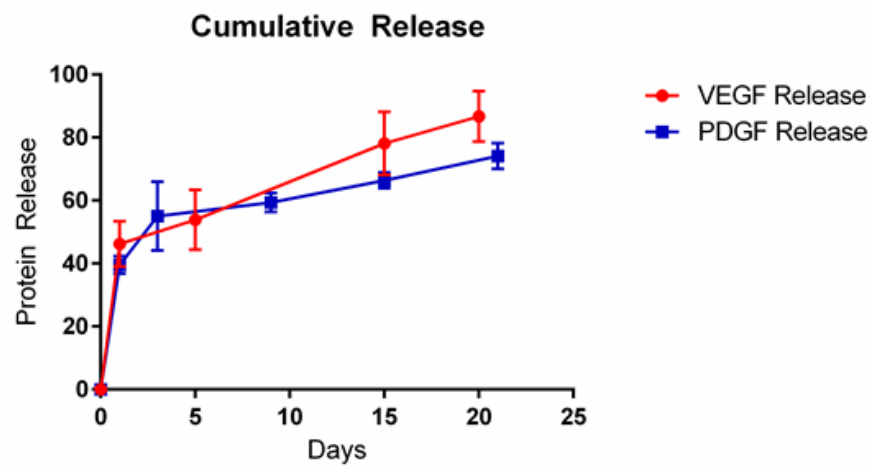

Figure 2. Heparin-binding proteins preferentially load into the coacervate (A) PDGF and VEGF exhibit loading efficiencies over 8 times greater than total protein loading due to their affinity to heparin. (B) In vitro release of VEGF and PDGF as representative PRP proteins show a sustained release over 3 weeks. Reported as mean \pm SD.

PRP coacervate accelerates pig wound closure

To assess in vivo efficacy, $20 \mathrm{~mm}$ round wounds were created on the backs of Yorkshire pigs and treated with PRP coacervate, an identical dose of free PRP, unloaded coacervate delivery vehicle alone, or saline as controls. Porcine skin, like that of humans, heals primarily by reepethelialization [34]. Immunofluorescent detection of cytokeratin for epidermal keratinocyte cells showed a significantly increased reepithelialization rate ( $35 \%$ relative to saline) 10 days after wounding compared to saline and delivery vehicle alone (Figure 3). No significant differences existed between other treatment groups. Wounds treated with PRP coacervate also exhibited a thicker epidermis adjacent to the wound margin compared to other 
groups. Positive cytokeratin staining was also observed in sebaceous glands and hair follicles of healthy tissue as expected.
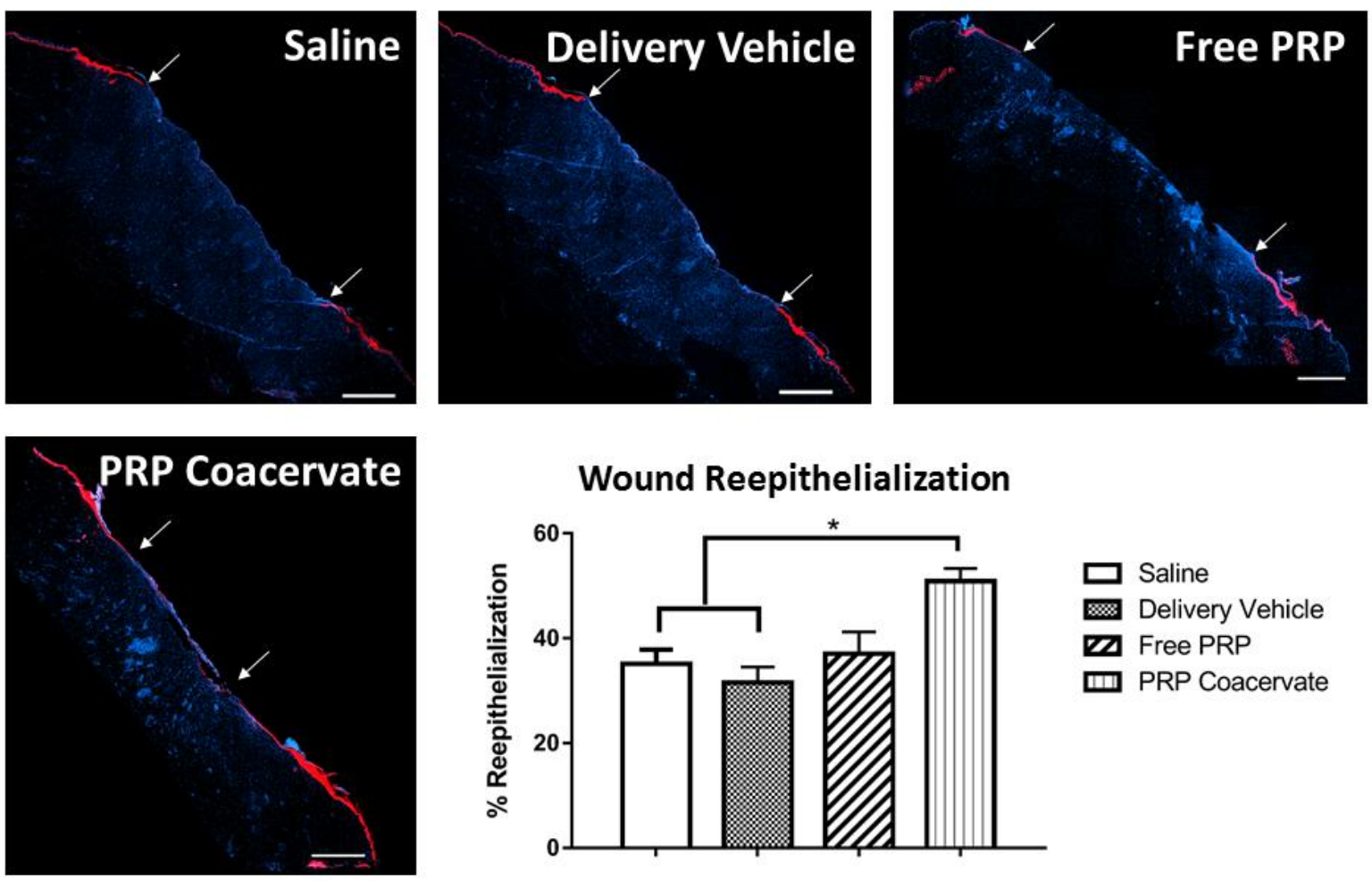

Figure 3. PRP coacervate accelerates reepithelialization of wounds

Wound sections showing coverage by keratinocytes 10 days after treatment. Wound coverage at ten days is shown relative to the 10-day wound length. Wounds treated with PRP coacervate exhibit a $35 \%$ increase in reepithelialization relative to saline while free PRP does not significantly improve reepithelialization. Data presented as mean \pm SEM. Scale bars are $2 \mathrm{~mm}$. * indicates $\mathrm{p}<0.05$ 
A
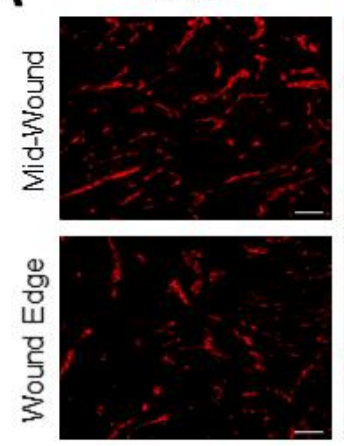

B

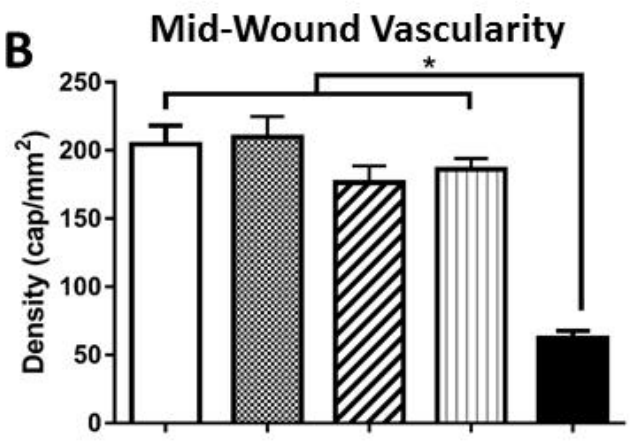

Vehicle
Delivery
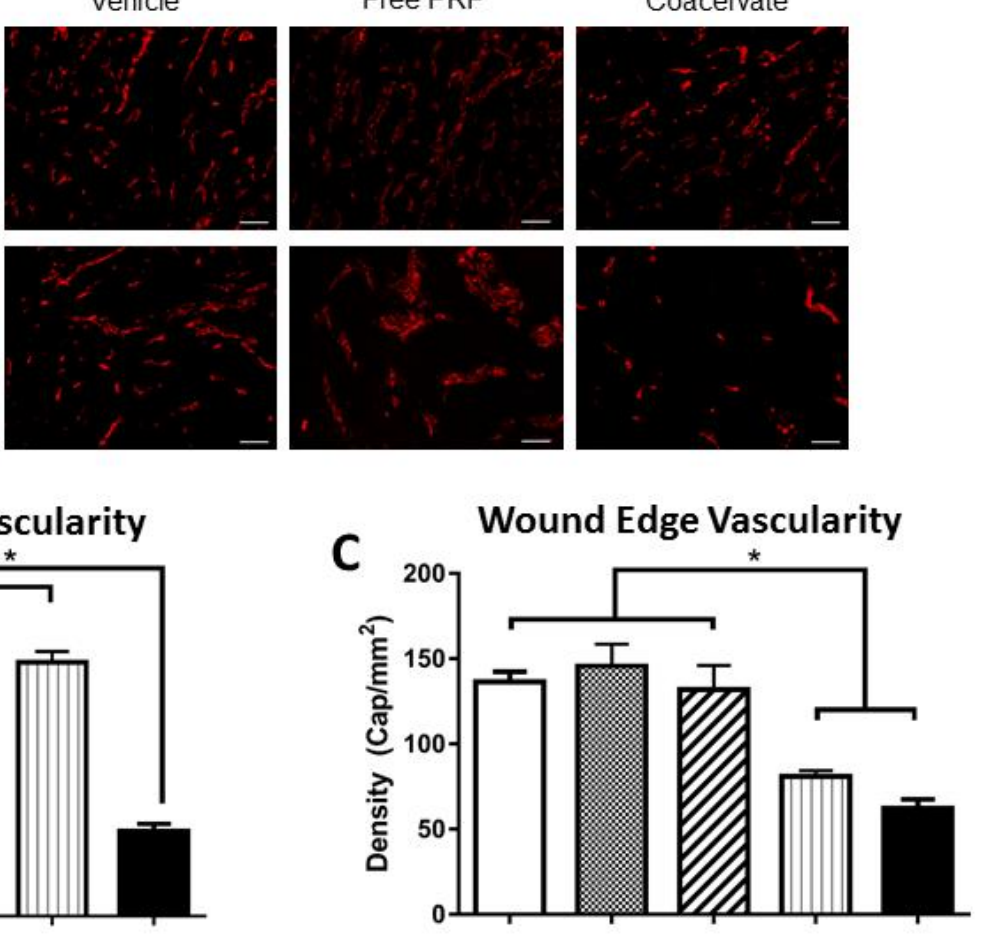

PRP

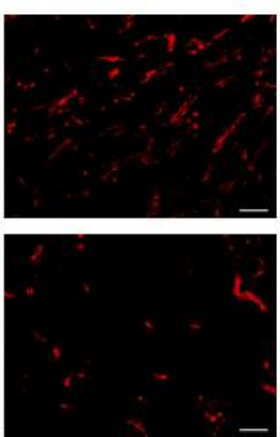

Saline Delivery Vehicle W Free PRP
$\square$ PRP Coacervate $\quad$ Healthy Tissue

Figure 4. PRP coacervate improves vascular maturity at the wound margin (A) Staining with vWF for endothelial cells shows a high vascularity within all wounds with the exception of PRP coacervate when imaged at the wound margin. (B) All wounds exhibit higher vascularity than healthy tissue mid-wound at 10 days. (C) When imaged at the wound margin, wound receiving PRP coacervate have similar vascular density to healthy tissue while all other wounds exhibit a significant increase in vascularity. Scale bars $=100 \mu \mathrm{m}$. ${ }^{*}$ indicates $p<0.05$

\section{PRP coacervate modulates vascular density}

The vascular density of the wounds was measured at day 10 using vWF immunofluorescence to identify endothelial cells. In the wound center, vascular density was significantly higher than in healthy tissue indicating an influx of blood vessels into the wound bed, and no significant differences between treatment groups were observed (Figure 4a,b). However, at the wound edges, blood vessel density of wounds treated with PRP coacervate resembled that of uninjured tissue (Figure 4a).

Furthermore, the blood vessel density in PRP coacervate treated wounds was 
significantly lower than all other treatment groups (Figure 4a,c). Since wounds heal more quickly at the edges, these data suggest that wounds treated with PRP coacervate were at an advanced stage of healing relative to other treatments.
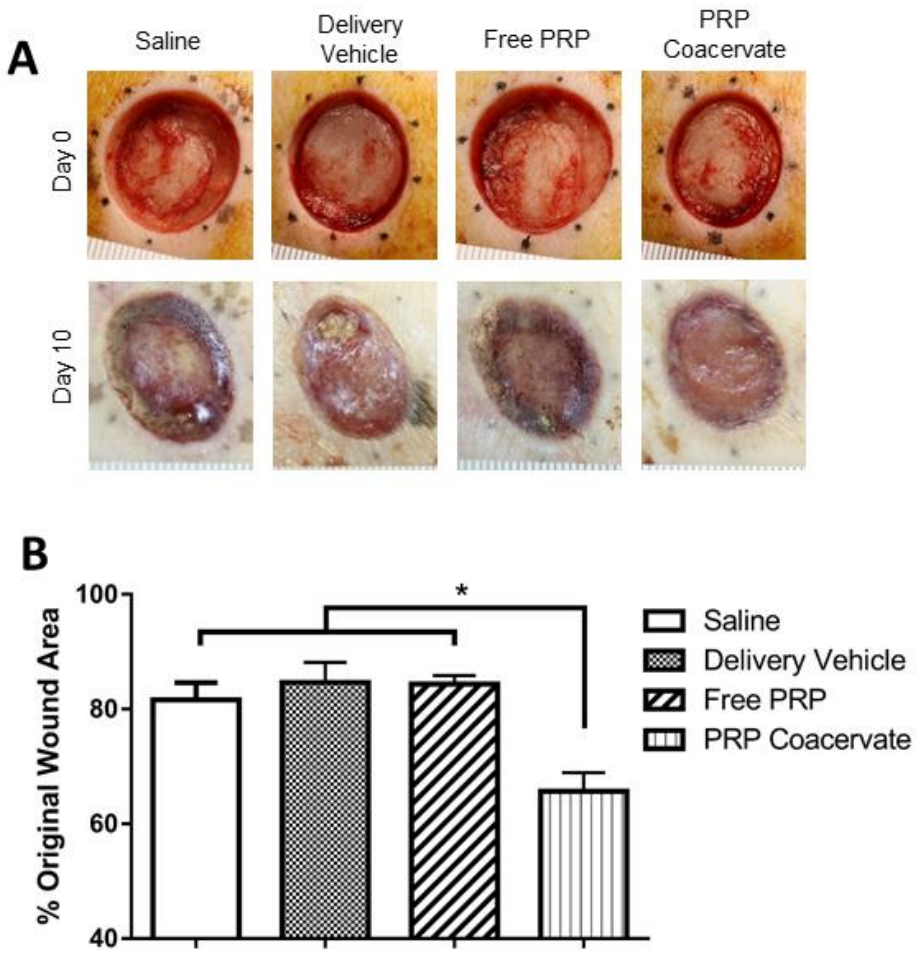

Figure 5. Controlled release of PRP reduces overall wound size

(A) Representative wound images show overall wound health at 10 days. All treatment groups other than PRP coacervate exhibit incomplete filling of the wound bed at this time point. Wounds receiving PRP coacervate were consistently a healthy pink color and smaller in area than other treatment groups. Tick marks are mm. (B) PRP coacervate shows significantly smaller wound size compared to all other treatments 10 days after wounding. * indicates $p<0.05$

\section{PRP coacervate decreases wound size}

Wound closure was also analyzed macroscopically to confirm measurements made histologically. Automated measurements of wound area showed a significant decrease in wound size after 10 days compared to all other treatments (65\% original wound size for PRP coacervate treated wounds compared to $83 \%$ when treated with saline). Free PRP or the delivery vehicle alone did not cause a significant reduction in wound size (Figure 5b). Further, wounds that did not receive PRP coacervate 
treatment were visibly deeper and dark red in color, indicating unhealthy granulation tissue formation (Figure 5a) [35]. The light pink color and raised appearance of granulation tissue seen in the PRP coacervate wounds indicate healthy granulation [35]. No signs of infection were present in any wound.

A
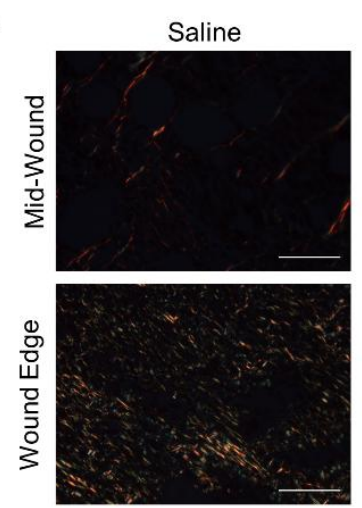

B

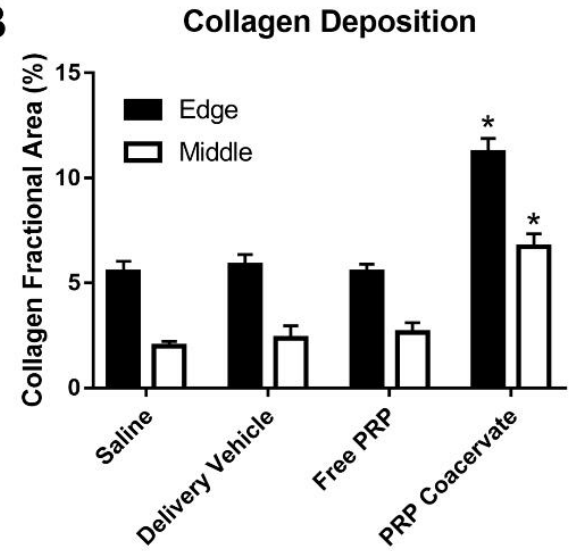

Delivery
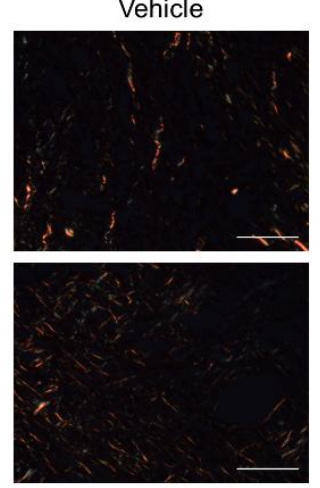

\begin{abstract}
C
\end{abstract}

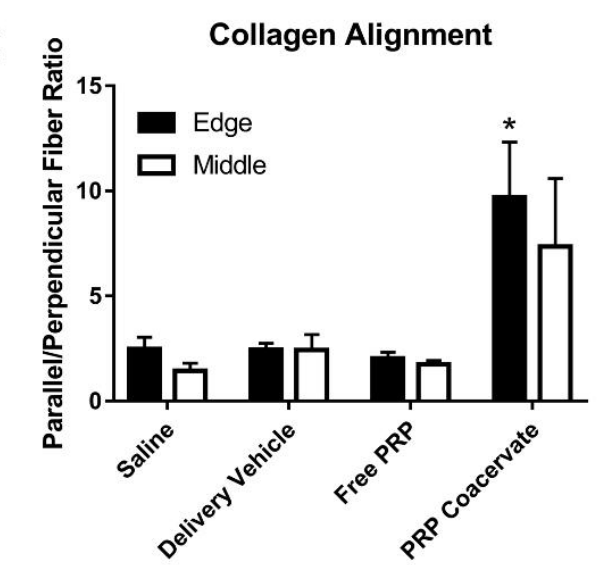

Figure 6. PRP coacervate increases collagen deposition and alignment

(A) Representative images of picrosirius red staining shows increased collagen content and alignment along the wound surface in response to PRP coacervate. The wound surface runs diagonally from top-left to bottom-right. (B) At both the wound edge and center, PRP coacervate significantly increases collagen content.

Regardless of treatment, collagen content was greater at the wound edge than center. (C) PRP coacervate significantly improves collagen alignment along the wound surface. This trend is also seen mid-wound. ${ }^{*}$ indicates $p<0.01$ from all other treatments in the corresponding location. Scale bars are $100 \mu \mathrm{m}$.

\section{PRP coacervate accelerates granulation tissue maturation}

Granulation tissue provides a temporary matrix for cells to infiltrate and repair the wound bed after injury. As expected, H\&E staining showed high cellularity and vascularity in the granulation tissue of all wounds relative to surrounding healthy 
tissue (Figure S1). Collagen deposition by fibroblasts had begun within 10 days in all wounds as seen by MTS (Figure S2). Collagen deposition and alignment was quantified the edge and middle of each wound to determine the effect of PRP coacervate on granulation tissue formation. In both the wound edges and center, it was evident that treatment with PRP coacervate resulted in increased collagen deposition (Figure 6a,b). Regardless of treatment, the amount of collagen was significantly greater at the wound edges than in the center. This is consistent with our analysis of wound angiogenesis which shows advanced healing at the wound edge. Collagen alignment was also quantified as the ratio of collagen fibers running parallel to the fibers running perpendicular to the wound surface. A higher ratio indicates preferential alignment along the wound surface, indicative of advanced scar formation; a ratio near 1 indicates no preferential alignment and therefore an earlier stage of granulation tissue organization. At the wound edge, PRP coacervate significantly increased collagen alignment parallel to the wound surface compared to all other treatments. This trend was also seen in the wound centers but was not significant (Figure 6a,c). These results demonstrate the ability of PRP coacervate to beneficially modulate collagen deposition and alignment in the early stages of wound granulation..

\section{Discussion}

The widespread use of PRP as a therapy remains highly debated in medicine. Autologous PRP is typically highly variable because of inherent differences between patients, methods of preparation, and whether it is used as a liquid or a thrombininduced hydrogel $[16,36]$. This leads to inconsistent results between patients and studies when used clinically as an autologous therapy. PRP activated by thrombin can be applied as a gel and has been described as a controlled release system to 
improve the efficacy of PRP [18]. However, these studies have been restricted to small animal models or large animals models without sufficient characterization of protein release, and most have not been injectable [37]. Here we report a systematic study of the effects of PRP in a porcine model and the benefits of controlled release for wound healing.

Appropriate controlled delivery systems are crucial to achieve high therapeutic efficacy of growth factors [2]. Short half-lives, adverse off-target effects, and poor spatio-temporal control are common issues of free protein application which can be solved using controlled release systems [38]. One intuitive approach is to harness the native properties of ECM molecules which sequester growth factors, prevent their degradation and promote their bioactivity [39]. Several different types of delivery platforms have been developed along this theme, utilizing ECM molecules vitronectin, fibronectin, and heparin. In one such platform, vitronectin complexes with insulin-like growth factor (IGF) and epidermal growth factor (EGF) demonstrated wound healing efficacy in large animals and safety in humans [40, 41]. In another approach, synthetic fibronectin-like peptides were developed to deliver several different heparin-binding growth factors and cytokines and evaluated in small animal studies [42-44]. Our lab has characterized a heparin-based platform which takes the form of liquid coacervate droplets that load and release heparin-binding proteins [1, 45]. HB-EGF delivered by the coacervate system accelerated healing in both diabetic and non-diabetic rodent wound models [24, 28].

The use of multiple growth factors with the coacervate system has been explored previously to stimulate angiogenesis with VEGF and hepatocyte growth factor (HGF) [46]. The co-release profile of these two factors was nearly linear over 3 weeks with no initial burst release. In comparison, both PDGF and VEGF exhibit a 
$50 \%$ burst release from the PRP coacervate within 1 day of coacervate formation, followed by a nearly linear release profile over 3 weeks (figure 2b). Interactions between heparin and growth factors are primarily charge-driven [20]. When using small doses of proteins as was done previously, this interaction has negligible effects on the PEAD:heparin charge-based interaction. However, this study utilizes high protein concentrations; as more proteins bind to a heparin molecule, fewer sulfate groups are available to bind the PEAD polycation. Thus the coacervate formed is less stable than those formed with small protein doses. This causes the coacervate to dissociate at a faster rate, which likely explains the high initial release of proteins shown here.

Heparin has been used in many growth factor therapies since the heparin:growth factor complex is stable and resistant to proteolysis [47, 48]. Additionally, heparin potentiates the bioactivity of the proteins by facilitating their reactions with cell surface receptors [39, 49]. Indeed, heparin also has several beneficial roles in the wound healing process [50]. Its use in our delivery system provides the advantage of preferentially loading heparin-binding growth factors for sustained release. Although less than $10 \%$ of the total protein content of PRP becomes incorporated into the coacervate, heparin-binding proteins such as PDGF and VEGF exhibited a loading efficiency exceeding $60 \%$. To our knowledge this is the first report of a PRP delivery system that is able to preferentially load and release heparin-binding growth factors from complex protein solutions. Prior studies utilizing heparin have largely involved its covalent immobilization to the surface of a polymeric scaffold or within a hydrogel, thereby endowing the biomaterial with growth factor affinity [51]. However, covalent linkage may partially or fully inhibit the activity of heparin, reducing growth factor loading efficiency or creating a steric selectivity for 
heparin-binding protein of certain sizes or conformations. The coacervate platform employs heparin in free-form which maintains its native functionality and consistently provides high loading capacity. Furthermore, the heparin-binding complexes are mobile and able to interact with cell surface receptors and thereby potentiate growth factor bioactivity. In comparison, immobilized heparin may hold growth factors to a surface or deep within a scaffold that cells must infiltrate to access. Although heparin is classically used as an anticoagulant, we have not observed excessive bleeding in wounds treated with the coacervate. This is likely because heparin is not free in our system but instead neutralized by virtue of being bound to growth factors and the polycation. A system using free heparin amphiphiles has also been reported, yet growth factor release was not characterized beyond 10 days [52, 53].The advantages of the coacervate system are owed to the unique liquid-liquid phase separated structure held together by electrostatic interactions of heparin and the novel cationic polymer PEAD.

A porcine model of full-thickness excisional wounds is one of the best preclinical wound healing models as pig skin heals by similar mechanisms to human patients [34]. Here we demonstrate that PRP itself is unable to improve wound healing; however the controlled release of its therapeutic proteins can accelerate the wound healing process. Wound vascularization and collagen fiber deposition and alignment within in the granulation tissue indicated improved wound maturation in PRP coacervate-treated wounds. Additionally, smaller gross wound size and enhanced reepithelialization shows that wound closure was also accelerated. The use of PRP coacervate essentially creates two stages of release: an initial diffusion of all unincorporated PRP proteins followed by a sustained release of the heparinbinding growth factors. Sequential release of individual growth factors by the 
coacervate has previously demonstrated enhanced therapeutic efficacy $[26,54]$. The PRP coacervate creates an analogous situation in the present study where high initial concentrations of unincorporated proteins induce a strong acute response followed by the sustained release of heparin-binding proteins which enact long-term effects. Additional studies are needed to determine the effect of incorporated versus unincorporated proteins within the PRP coacervate therapy.

Angiogenesis is a vital component of the wound healing process. The formation of leaky capillaries allows cells and proteins to infiltrate the damaged tissue and begin healing $[5,55]$. A high capillary density was observed in the center of all wounds assessed in this study, indicating the normal angiogenic process was not significantly disturbed. Since wounds heal from the edge, granulation tissue near the wound edge matures faster than the center of the wound where granulation tissue forms last [55]. Therefore we are able to see a progression of the angiogenic process moving from the edge to the interior of the wound. The vessel density of all treatment groups was lower at the wound perimeter compared to the center, indicating that the wound margin had progressed through the peak of angiogenesis and was returning to the vascularization levels of native skin. These regional differences in healing based have been established in several previous studies, particularly regarding the angiogenic response $[56,57]$. The difference arises in wounds treated with PRP coacervate, where vessel density is similar to other wounds at the center but is significantly lower at the wound margin. This suggests that controlled PRP release accelerates the angiogenic phase of healing, returning vessel density to near-normal levels at a faster rate and exhibiting a more advanced stage of healing. Longer timepoints are necessary to confirm that acceleration of the angiogenic phase 
improves long-term healing of these wounds. This has significant relevance when comorbidities are present such as peripheral arterial disease or diabetes.

There are several limitations of the current study to be addressed in the future. The relatively short duration (10 days) of this study allows insight into the early stages of wound healing such as reepithelialization rate, changes in the angiogenic response, and collagen deposition. We have shown changes in these parameters in response to PRP coacervate, but now longer timepoints are needed to assess functional outcomes such as full reepithelialization, wound maturation, and the mechanical strength of the resulting scar. Due to a limited sample size, we were unable to determine if PRP coacervate follows a dose-dependent outcome, but in future studies we will optimize PRP dosing for wound healing. Additionally, we plan to employ diabetic pig models to evaluate the efficacy of PRP coacervate for chronic wound healing.

This study demonstrates the therapeutic benefits of controlled PRP release for wound healing applications. A quick and simple assembly method allows adaptability for either rapid autologous preparation at the bedside or an allogenic sourced off-theshelf product. The liquid nature of our coacervate system allows it to be injected directly into the wound bed or incorporated into a substrate for application. This is especially advantageous as it can be applied to commercially available sponges containing antibiotics to help prevent infection. Other systems are not as compatible with the current standard-of-care, making it difficult to both treat the wound and prevent infection simultaneously. This makes the coacervate system easily adaptable to a number of different dressings and bandages. The controlled release mechanism allows treatment to be applied once every few weeks rather than daily as seen in traditional protein therapies. Given the ability of this therapy to capture many 
proteins, this study suggests that the coacervate vehicle could also be adapted to other therapeutic applications in large animal models.

\section{Conclusions}

This study evaluated the efficacy of traditional PRP treatment and the advantage of a controlled release PRP formulation on cutaneous wound healing. We have shown that the sustained release of PRP proteins is able to significantly improve wound closure within 10 days of wounding, while the clinical standard of free PRP proteins demonstrates no significant benefit. This is accompanied by a significant alteration in vascularity of the wound edge, returning vessel density to near-healthy levels. These results suggest that the controlled delivery of PRP proteins using the coacervate vehicle encourages accelerated healing of cutaneous wounds in a porcine model. The widespread use of PRP in humans combined with the clinical relevance of the porcine wound healing model emphasize the impact of this study on the translational potential to enhance autologous procedures with an easy-to-use protein delivery platform

\section{Acknowledgements}

The authors would like to thank Dr. Alan Wells for offering insight on analysis methods and Stacy Lee Cashman for providing her animal care expertise throughout this project. Additionally, we would like to thank Angela Urban and Jai Vekaria for their assistance in animal care. This work was supported in part by a P3HVB seed grant from the Pittsburgh Heart, Lung and Blood Vascular Medicine Institute, the National Institute of Nursing Research (1R01NR016436-01), and the National Institutes of Health Biomechanics in Regenerative Medicine T32 training program (grant number ST32EB003392-09). We thank Scientific Protein Labs for providing heparin. 


\section{References}

[1] H. Chu, N.R. Johnson, N.S. Mason, Y. Wang, A [polycation:heparin] complex releases growth factors with enhanced bioactivity, Journal of controlled release : official journal of the Controlled Release Society, 150 (2011) 157-163.

[2] N.R. Johnson, Y. Wang, Drug delivery systems for wound healing, Current pharmaceutical biotechnology, 16 (2015) 621-629.

[3] K. Lee, E.A. Silva, D.J. Mooney, Growth factor delivery-based tissue engineering: general approaches and a review of recent developments, Journal of the Royal Society, Interface / the Royal Society, 8 (2011) 153-170.

[4] E. Anitua, M.H. Alkhraisat, G. Orive, Perspectives and challenges in regenerative medicine using plasma rich in growth factors, Journal of Controlled Release, 157 (2012) 29-38.

[5] S.A. Eming, P. Martin, M. Tomic-Canic, Wound repair and regeneration: mechanisms, signaling, and translation, Sci Transl Med, 6 (2014) 265sr266.

[6] C.K. Sen, G.M. Gordillo, S. Roy, R. Kirsner, L. Lambert, T.K. Hunt, F. Gottrup, G.C. Gurtner, M.T. Longaker, Human skin wounds: a major and snowballing threat to public health and the economy, Wound Repair Regen, 17 (2009) 763-771.

[7] R.C. Fang, R.D. Galiano, A review of becaplermin gel in the treatment of diabetic neuropathic foot ulcers, Biologics : targets \& therapy, 2 (2008) 1-12.

[8] X.H. Zhao, H.F. Gu, Z.R. Xu, Q. Zhang, X.Y. Lv, X.J. Zheng, Y.M. Yang, Efficacy of topical recombinant human platelet-derived growth factor for treatment of diabetic lower-extremity ulcers: Systematic review and meta-analysis, Metabolism: clinical and experimental, 63 (2014) 1304-1313.

[9] N. Papanas, E. Maltezos, Becaplermin gel in the treatment of diabetic neuropathic foot ulcers, Clinical interventions in aging, 3 (2008) 233-240.

[10] J.R. Hanft, R.A. Pollak, A. Barbul, C. van Gils, P.S. Kwon, S.M. Gray, C.J. Lynch, C.P. Semba, T.J. Breen, Phase I trial on the safety of topical rhVEGF on chronic neuropathic diabetic foot ulcers, Journal of wound care, 17 (2008) 30-32, 34-37.

[11] B. Ma, D.S. Cheng, Z.F. Xia, D.F. Ben, W. Lu, Z.F. Cao, Q. Wang, J. He, J.K. Chai, C.A. Shen, Y.H. Sun, G.A. Zhang, X.H. Hu, Randomized, multicenter, double-blind, and placebo-controlled trial using topical recombinant human acidic fibroblast growth factor for deep partial-thickness burns and skin graft donor site, Wound repair and regeneration : official publication of the Wound Healing Society [and] the European Tissue Repair Society, 15 (2007) 795-799.

[12] M.W. Ferguson, J. Duncan, J. Bond, J. Bush, P. Durani, K. So, L. Taylor, J. Chantrey, T. Mason, G. James, H. Laverty, N.L. Occleston, A. Sattar, A. Ludlow, S. O'Kane, Prophylactic administration of avotermin for improvement of skin scarring: three double-blind, placebo-controlled, phase I/II studies, Lancet, 373 (2009) 1264-1274.

[13] F. Mussano, T. Genova, L. Munaron, S. Petrillo, F. Erovigni, S. Carossa, Cytokine, chemokine, and growth factor profile of platelet-rich plasma, Platelets, (2016) 1-5.

[14] Z.Y. Zhang, A.W. Huang, J.J. Fan, K. Wei, D. Jin, B. Chen, D. Li, L. Bi, J. Wang, G. Pei, The potential use of allogeneic platelet-rich plasma for large bone defect treatment: immunogenicity and defect healing efficacy, Cell Transplant, 22 (2013) 175-187.

[15] V.R. Driver, J. Hanft, C.P. Fylling, J.M. Beriou, G. Autologel Diabetic Foot Ulcer Study, A prospective, randomized, controlled trial of autologous platelet-rich plasma gel for the treatment of diabetic foot ulcers, Ostomy/wound management, 52 (2006) 68-70, 72, 74 passim.

[16] K.K. Middleton, V. Barro, B. Muller, S. Terada, F.H. Fu, Evaluation of the effects of platelet-rich plasma (PRP) therapy involved in the healing of sports-related soft tissue injuries, lowa Orthop J, 32 (2012) 150-163.

[17] T.D. Vu, S.N. Pal, L.K. Ti, E.C. Martinez, A.J. Rufaihah, L.H. Ling, C.N. Lee, A.M. Richards, T. Kofidis, An autologous platelet-rich plasma hydrogel compound restores left ventricular structure, function and ameliorates adverse remodeling in a minimally invasive large animal myocardial restoration model: a translational approach: Vu and Pal "Myocardial Repair: PRP, Hydrogel and Supplements", Biomaterials, 45 (2015) 27-35. 
[18] H.S. Yang, J. Shin, S.H. Bhang, J.Y. Shin, J. Park, G.I. Im, C.S. Kim, B.S. Kim, Enhanced skin wound healing by a sustained release of growth factors contained in platelet-rich plasma, Exp Mol Med, 43 (2011) 622-629.

[19] M.J. Martinez-Zapata, A.J. Marti-Carvajal, I. Sola, J.A. Exposito, I. Bolibar, L. Rodriguez, J. Garcia, Autologous platelet-rich plasma for treating chronic wounds, The Cochrane database of systematic reviews, 10 (2012) Cd006899.

[20] I. Capila, R.J. Linhardt, Heparin-protein interactions, Angewandte Chemie, 41 (2002) 391-412. [21] L. Pellegrini, D.F. Burke, F. von Delft, B. Mulloy, T.L. Blundell, Crystal structure of fibroblast growth factor receptor ectodomain bound to ligand and heparin, Nature, 407 (2000) 1029-1034. [22] H. Chu, J. Gao, Y. Wang, Design, synthesis, and biocompatibility of an arginine-based polyester, Biotechnology progress, 28 (2012) 257-264.

[23] W.C. Chen, B.G. Lee, D.W. Park, K. Kim, H. Chu, K. Kim, J. Huard, Y. Wang, Controlled dual delivery of fibroblast growth factor-2 and Interleukin-10 by heparin-based coacervate synergistically enhances ischemic heart repair, Biomaterials, 72 (2015) 138-151.

[24] N.R. Johnson, Y. Wang, Controlled delivery of heparin-binding EGF-like growth factor yields fast and comprehensive wound healing, Journal of controlled release : official journal of the Controlled Release Society, 166 (2013) 124-129.

[25] H. Chu, C.W. Chen, J. Huard, Y. Wang, The effect of a heparin-based coacervate of fibroblast growth factor-2 on scarring in the infarcted myocardium, Biomaterials, 34 (2013) 1747-1756.

[26] H.K. Awada, N.R. Johnson, Y. Wang, Sequential delivery of angiogenic growth factors improves revascularization and heart function after myocardial infarction, Journal of controlled release : official journal of the Controlled Release Society, 207 (2015) 7-17.

[27] N.R. Johnson, Y. Wang, Controlled delivery of sonic hedgehog morphogen and its potential for cardiac repair, PloS one, 8 (2013) e63075.

[28] N.R. Johnson, Y. Wang, Coacervate delivery of HB-EGF accelerates healing of type 2 diabetic wounds, Wound Repair Regen, 23 (2015) 591-600.

[29] N.R. Johnson, Y. Wang, Controlled Delivery of Sonic Hedgehog with a Heparin-Based Coacervate, Methods in molecular biology, 1322 (2015) 1-7.

[30] J. Wu, J. Ye, J. Zhu, Z. Xiao, C. He, H. Shi, Y. Wang, C. Lin, H. Zhang, Y. Zhao, X. Fu, H. Chen, X. Li, L. $\mathrm{Li}$, J. Zheng, J. Xiao, Heparin-Based Coacervate of FGF2 Improves Dermal Regeneration by Asserting a Synergistic Role with Cell Proliferation and Endogenous Facilitated VEGF for Cutaneous Wound Healing, Biomacromolecules, 17 (2016) 2168-2177.

[31] C.H. Kuo, L. Leon, E.J. Chung, R.T. Huang, T.J. Sontag, C.A. Reardon, G.S. Getz, M. Tirrell, Y. Fang, Inhibition of atherosclerosis-promoting microRNAs via targeted polyelectrolyte complex micelles, Journal of materials chemistry. B, Materials for biology and medicine, 2 (2014) 8142-8153.

[32] D. Brett, A Review of Collagen and Collagen-based Wound Dressings, Wounds : a compendium of clinical research and practice, 20 (2008) 347-356.

[33] M. Laufer, C. Ashkenazi, D. Katz, M. Wolman, Orientation of collagen in wound healing, British journal of experimental pathology, 55 (1974) 233-236.

[34] T.P. Sullivan, W.H. Eaglstein, S.C. Davis, P. Mertz, The pig as a model for human wound healing, Wound Repair Regen, 9 (2001) 66-76.

[35] J.E. Grey, S. Enoch, K.G. Harding, Wound assessment, Bmj, 332 (2006) 285-288.

[36] S. Arora, V. Doda, U. Kotwal, M. Dogra, Quantification of platelets and platelet derived growth factors from platelet-rich-plasma (PRP) prepared at different centrifugal force (g) and time, Transfusion and apheresis science : official journal of the World Apheresis Association : official journal of the European Society for Haemapheresis, 54 (2016) 103-110.

[37] Y. Yan, D.L. Larson, Acceleration of Full-thickness Wound Healing in Porcine Model by Autologous Platelet Gel, Wounds : a compendium of clinical research and practice, 19 (2007) 79-86. [38] P. Koria, Delivery of growth factors for tissue regeneration and wound healing, BioDrugs, 26 (2012) 163-175.

[39] G.S. Schultz, A. Wysocki, Interactions between extracellular matrix and growth factors in wound healing, Wound Repair Regen, 17 (2009) 153-162. 
[40] Y. Xie, Z. Upton, S. Richards, S.C. Rizzi, D.I. Leavesley, Hyaluronic acid: Evaluation as a potential delivery vehicle for vitronectin:growth factor complexes in wound healing applications, J Control Release, (2011).

[41] Z. Upton, H.J. Wallace, G.K. Shooter, D.R. van Lonkhuyzen, S. Yeoh-Ellerton, E.A. Rayment, J.M. Fleming, D. Broszczak, D. Queen, R.G. Sibbald, D.I. Leavesley, M.C. Stacey, Human pilot studies reveal the potential of a vitronectin: growth factor complex as a treatment for chronic wounds, International wound journal, 8 (2011) 522-532.

[42] M.M. Martino, F. Tortelli, M. Mochizuki, S. Traub, D. Ben-David, G.A. Kuhn, R. Muller, E. Livne, S.A. Eming, J.A. Hubbell, Engineering the growth factor microenvironment with fibronectin domains to promote wound and bone tissue healing, Sci Transl Med, 3 (2011) 100ra189.

[43] F. Tortelli, M. Pisano, P.S. Briquez, M.M. Martino, J.A. Hubbell, Fibronectin binding modulates CXCL11 activity and facilitates wound healing, PLoS One, 8 (2013) e79610.

[44] M.M. Martino, P.S. Briquez, A. Ranga, M.P. Lutolf, J.A. Hubbell, Heparin-binding domain of fibrin(ogen) binds growth factors and promotes tissue repair when incorporated within a synthetic matrix, Proc Natl Acad Sci U S A, 110 (2013) 4563-4568.

[45] N.R. Johnson, T. Ambe, Y. Wang, Lysine-based polycation:heparin coacervate for controlled protein delivery, Acta biomaterialia, 10 (2014) 40-46.

[46] H.K. Awada, N.R. Johnson, Y. Wang, Dual delivery of vascular endothelial growth factor and hepatocyte growth factor coacervate displays strong angiogenic effects, Macromolecular bioscience, 14 (2014) 679-686.

[47] H. Chu, J. Gao, C.W. Chen, J. Huard, Y. Wang, Injectable fibroblast growth factor-2 coacervate for persistent angiogenesis, Proceedings of the National Academy of Sciences of the United States of America, 108 (2011) 13444-13449.

[48] L. Pellegrini, Role of heparan sulfate in fibroblast growth factor signalling: a structural view, Current opinion in structural biology, 11 (2001) 629-634.

[49] R.O. Hynes, The extracellular matrix: not just pretty fibrils, Science, 326 (2009) 1216-1219.

[50] P. Olczyk, L. Mencner, K. Komosinska-Vassev, Diverse Roles of Heparan Sulfate and Heparin in Wound Repair, BioMed research international, 2015 (2015) 549417.

[51] S.E. Sakiyama-Elbert, Incorporation of heparin into biomaterials, Acta Biomater, 10 (2014) 15811587.

[52] S.S. Lee, B.J. Huang, S.R. Kaltz, S. Sur, C.J. Newcomb, S.R. Stock, R.N. Shah, S.I. Stupp, Bone regeneration with low dose BMP-2 amplified by biomimetic supramolecular nanofibers within collagen scaffolds, Biomaterials, 34 (2013) 452-459.

[53] K. Rajangam, H.A. Behanna, M.J. Hui, X. Han, J.F. Hulvat, J.W. Lomasney, S.I. Stupp, Heparin binding nanostructures to promote growth of blood vessels, Nano Lett, 6 (2006) 2086-2090.

[54] H.K. Awada, L.A. Johnson, T.K. Hitchens, L.M. Foley, Y. Wang, Factorial Design of Experiments to Optimize Multiple Protein Delivery for Cardiac Repair, ACS Biomaterials Science \& Engineering, 2 (2016) 879-886.

[55] T. Velnar, T. Bailey, V. Smrkolj, The wound healing process: an overview of the cellular and molecular mechanisms, J Int Med Res, 37 (2009) 1528-1542.

[56] D.G. Greenhalgh, The role of apoptosis in wound healing, The international journal of biochemistry \& cell biology, 30 (1998) 1019-1030.

[57] M.G. Tonnesen, X. Feng, R.A. Clark, Angiogenesis in wound healing, The journal of investigative dermatology. Symposium proceedings, 5 (2000) 40-46. 


\section{Heparin PEAD Coacervate}

PRP
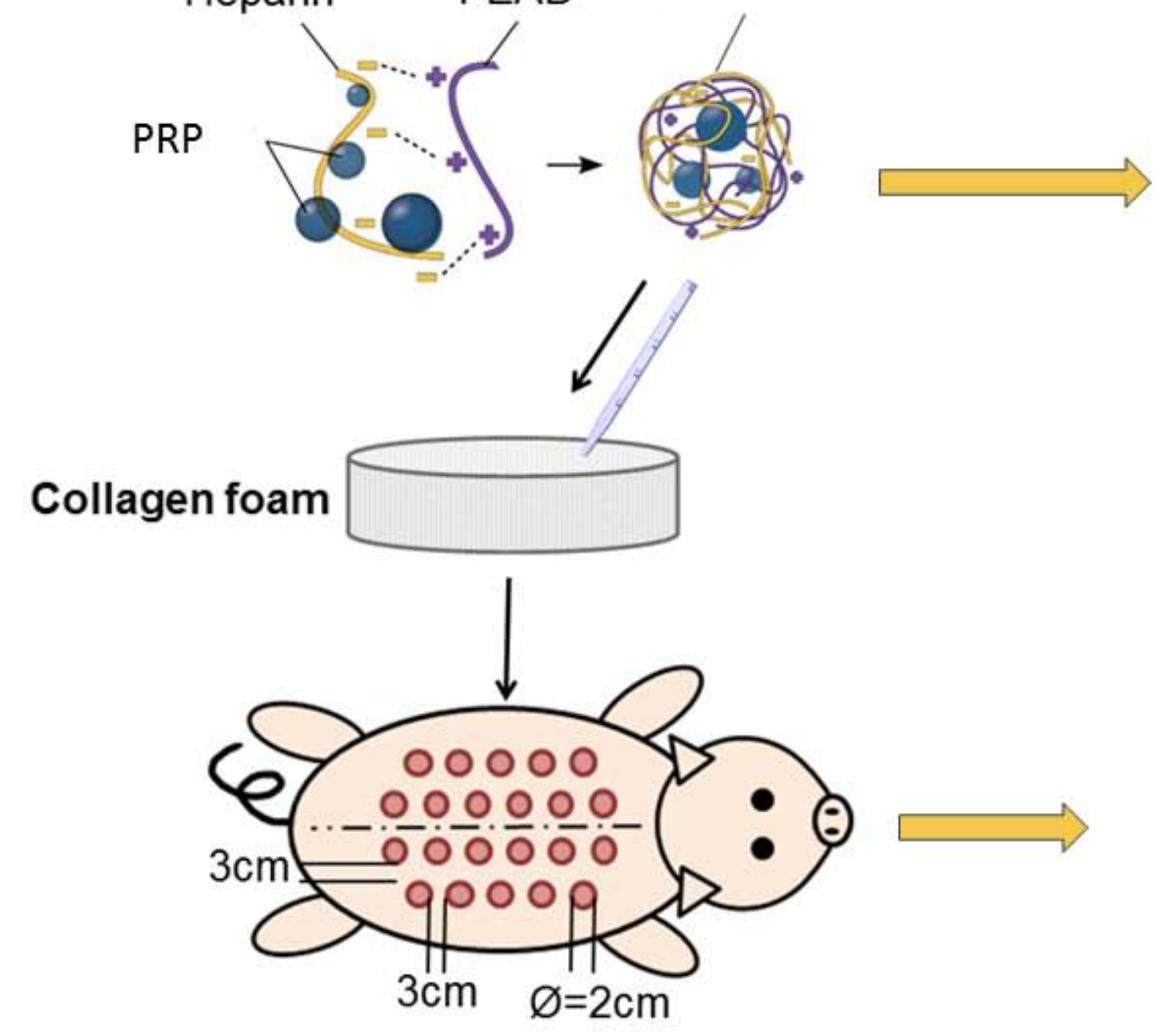

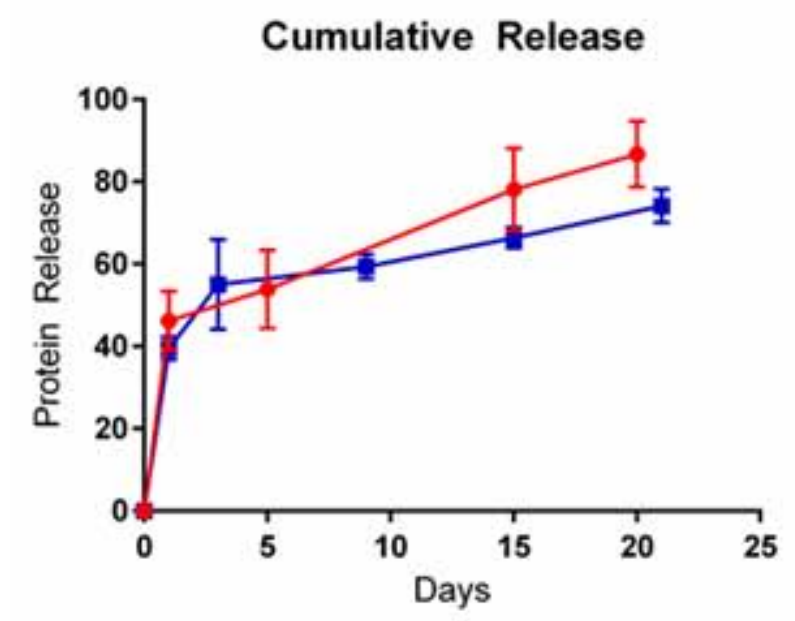

$\rightarrow$ VEGF Release

$\rightarrow$ PDGF Release

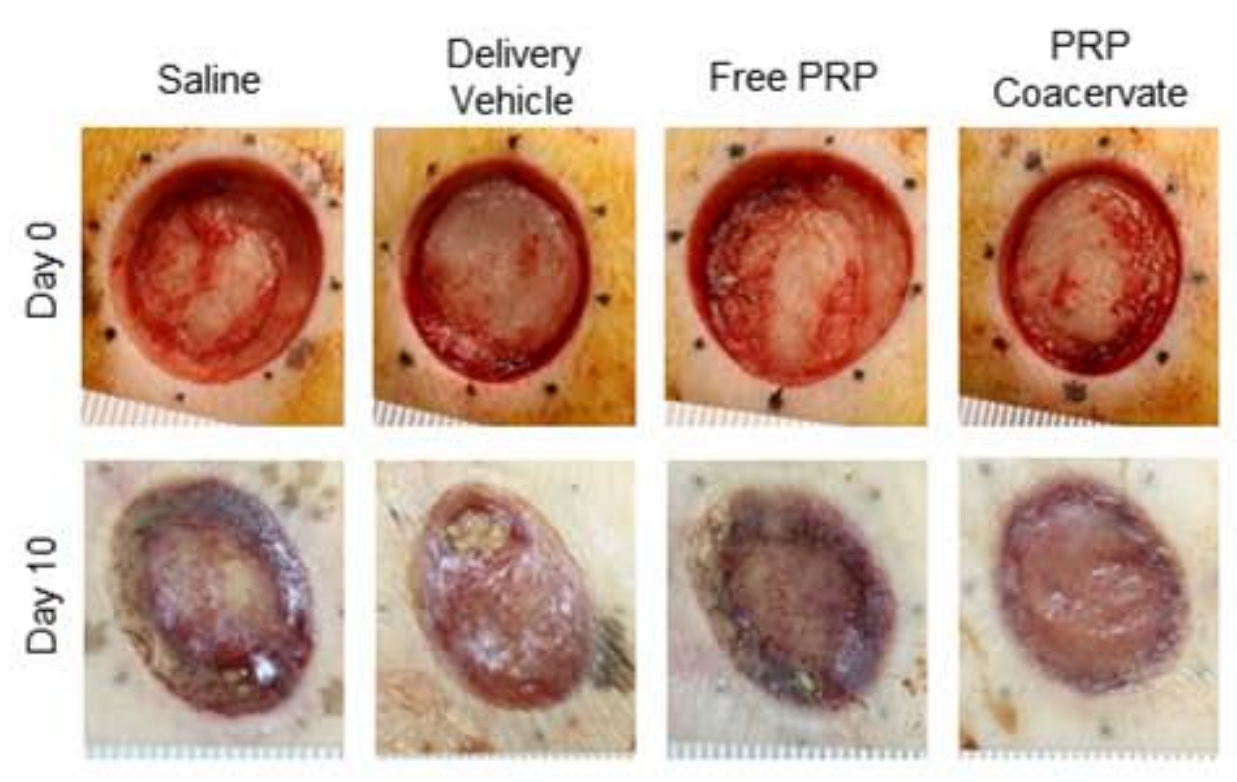

